\title{
Systems biology (un)certainties
}

\author{
P. D. W. Kirk ${ }^{1 * \dagger}$, A. C. Babtie ${ }^{2 * \dagger}$, M. P. H. Stumpf $f^{2 * \dagger}$ \\ ${ }^{1}$ MRC Biostatistics Unit, \\ Cambridge Institute of Public Health, CB2 OSR, UK \\ ${ }^{2}$ Centre for Integrative Systems Biology and Bioinformatics, \\ Imperial College London, SW7 2AZ, UK
}

*To whom correspondence should be addressed; E-mail: a.babtie@imperial.ac.uk, paul.kirk@mrc-bsu.cam.ac.uk, m.stumpf@imperial.ac.uk.

\section{Modelers must restore confidence in Systems and Computational Biology by avoiding over-interpretation of mathematical models and providing adequate assessments of uncertainty.}

Systems Biology, some have claimed (1), is attempting the impossible and is doomed to fail. Possible definitions abound, but Systems Biology is widely understood (including here) to be an approach for studying the behavior of systems of interacting biological components, that relies on combining experiments with computational and mathematical reasoning. Modeling complex systems occurs throughout the sciences, so it is perhaps not immediately clear why it should attract greater controversy in molecular and cell biology than elsewhere. We contend that the way in which models are often presented and (over) interpreted in the literature is at least partly to blame. As with experimental results, the key to successfully reporting a mathematical model is an honest appraisal and representation of uncertainty: in the models predictions, parameters, and (where appropriate) in the structure of the model itself. Deriving 
biological models is rarely straightforward. Although biology is, of course, subject to the same fundamental physical laws e.g. conservation of mass, energy and momentum as the other sciences, these often do not provide a good starting point for understanding how biological organisms and systems work. Biological modeling instead emphasizes context-specific levels of abstraction and relies upon experimental observations to decide if a particular model is useful. Typically, not all terms in a biological model are known or observable directly, and except for some highly specific systems it is impossible to measure the abundances of all the key players (molecules, cells or individuals) simultaneously and continuously. Thus, despite being often overlooked, the challenge is not only to identify descriptions and mathematical representations that provide insight, but also to communicate the inevitable uncertainty in the models (possibly many) unknowns. Although some authors have strongly advocated substituting these unknowns for estimates obtained from exogenous experimental assays (2), this is (a) often impossible, (b) rarely enough (since these estimates are themselves subject to considerable uncertainty), and (c) missing an opportunity to extract this information from the endogenous experimental data. In this context, Bayesian inference procedures (3), which naturally permit the integration of external prior knowledge or beliefs with newly observed data, may provide the most natural framework for expressing and reporting uncertainty. The number of unknowns in a model is partly determined by the scale of the system being studied. A biological system may range in scale from a few interacting molecules to whole populations of organisms, and this can have a huge impact on both the modeling approach and the associated assessment of uncertainty. For small systems, it might be possible to rely solely on strong prior knowledge and specify a model structure that reflects known interactions. For larger systems, automated network inference algorithms have been employed $(4,5)$. Such data-driven, hypothesis-generating approaches are often opposed, on the basis that the complexity of the biological system will render impossible the inverse problem of learning the true and complete underlying network (1). However, 
this opposition seems to be predicated upon misconceptions of the aims of large-scale network inference, which are typically to highlight and explore dependencies in datasets and thereby help generate new hypotheses worthy of further investigation rather than to uncover a single grand unifying model of the system. Unfortunately, not all network inference algorithms are equally effective, and the most popular approach using the correlation among expression levels of genes (6) results in a particularly poor basis on which to base further studies, let alone mechanistic models. A host of alternative approaches exist $(4,5)$ that provide better, more robust candidate networks, and which can incorporate expert or domain knowledge. Although large-scale grand unifying models have occasionally been sought (e.g. whole-cell models (7)), these efforts remain the exception. Many challenges remain for models of such scope, including how to validate their quality and adequately report the uncertainty in both their overall global structure, and their implied submodels. Whatever the scale of the system, we have to avoid over-interpretation by remaining aware of the limitations of our models, and steer clear of the fallacy that if it fits the data, it must be the truth. For any dataset, many different models are likely to provide plausible fits, while still remaining consistent with current knowledge. Even for a small-scale (5-gene) regulatory network, it can be possible to find tens of thousands of models that provide qualitatively perfect fits even to dense, low noise datasets, but which yield a variety of (often contradictory) insights into the regulatory relationships between genes (8). Assessing robustness of predictions and inferences across multiple alternative models can therefore be illuminating $(8-10)$, provided we keep in mind that our conclusions still depend on the particular set of models that we specify and are influenced by the experiments we choose to perform $(11,12)$. Crucial to remember is that models should not be expected to work in every conceivable context, and the most exciting results are frequently those that break existing models. Model uncertainty, as characterized by the ability to identify multiple models that explain current observations, therefore challenges us to design maximally discriminative experiments 
that will break as many models as possible $(12,13)$. For example, we have found that doseresponse curves typically provide too little discriminatory power, whereas carefully designed time-resolved analyses allow us to study even complicated models, e.g. in the context of proteasomal dynamics (14). Many of the criticisms of model development in Systems Biology stem from a lack of appreciation of the variety of roles that can be played by mathematical modeling (1). Such antipathy is partly driven by overstating implications and consequences of models, perhaps due to poor understanding of statistical learning and the value of reporting uncertainties. Frequent fallacies and bad practices continue to thrive, including the use of correlation to capture causal relationships, failure to address multiple testing problems, lack of confidence sets for parameters and models, and many more. The fact that models are simplified (but not simplistic) representations of real systems is precisely the property that makes them attractive to explore the consequences of our assumptions, and identify where we lack understanding of the principles governing a biological system. We should start to think of models as tools to uncover mechanisms that cannot be directly observed, akin to microscopes or NMR machines (15). Used and interpreted appropriately, with due attention paid to inherent uncertainties, the mathematical and computational modeling of biological systems allows us to explore hypotheses and learn about nature. But the relevance of these models depends on our ability to assess, understand, communicate and, ultimately cherish their uncertainties.

\section{References and Notes}

1. S. Brenner, Philosophical transactions of the Royal Society of London. Series B, Biological sciences 365, 207 (2010).

2. E. U. Azeloglu, R. Iyengar, Science signaling 8, fs8 (2015).

3. I. G. Johnston, et al., eLife 4, e07464 (2015). 


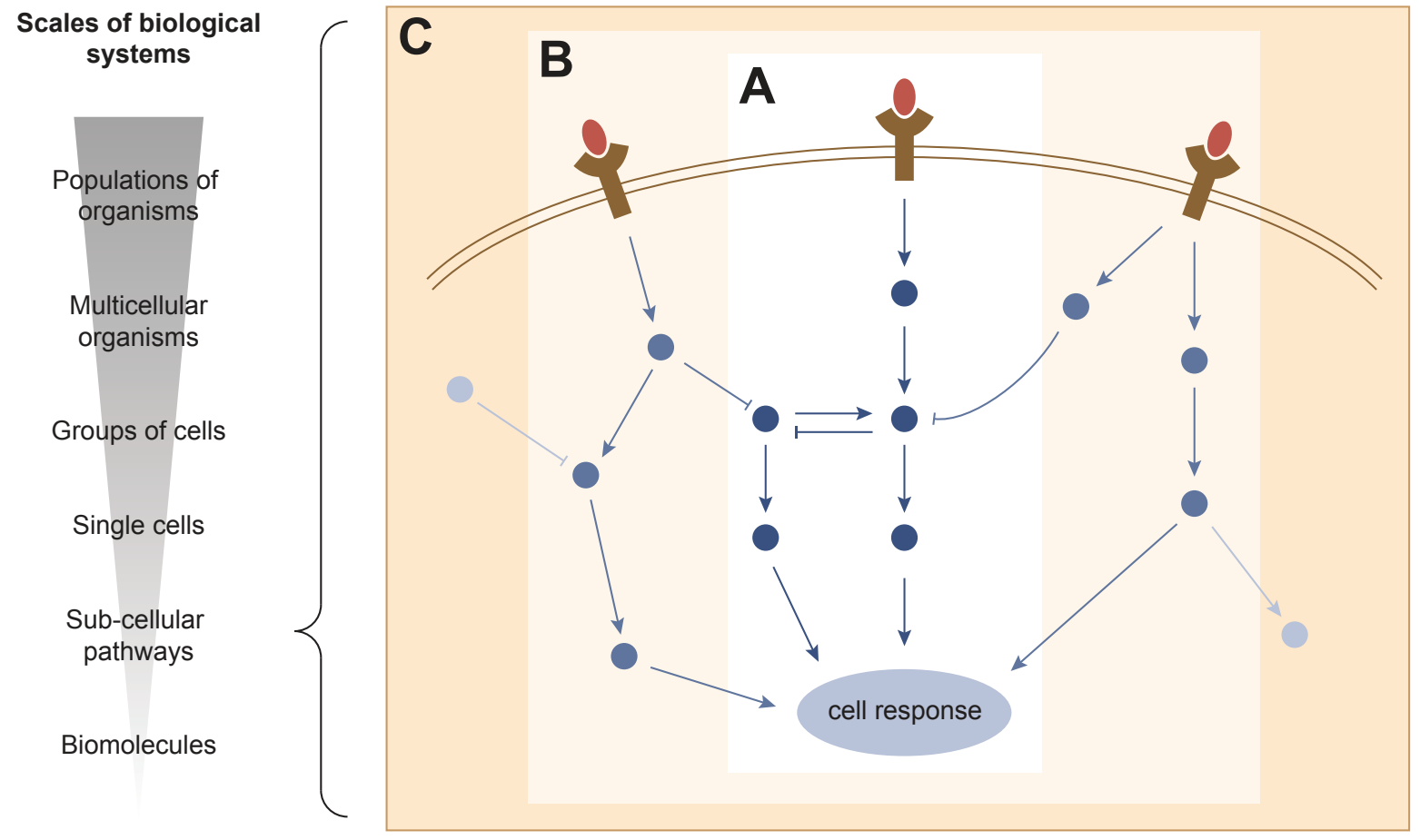

Figure 1: Mathematical models usually represent elements of a biological system at one scale. Systems biologists frequently focus on cellular and subcellular scale systems. Choosing the appropriate degree of abstraction and simplification can be influenced by current knowledge about the system, the quality and quantity of experimental data, the computational demands of a particular modeling approach, and the modeling aims. For example, when studying a signaling pathway, one must decide which biomolecules and interactions to include in a model. The scope of the model may be restricted to a single pathway or it might include the influence of parts of a wider interconnected network of signaling pathways. 
4. C. A. Penfold, D. L. Wild, Interface Focus 1, 857 (2011).

5. C. J. Oates, R. Amos, S. E. F. Spencer, Statistical applications in genetics and molecular biology 13, 611 (2014).

6. S.-J. Dunn, G. Martello, B. Yordanov, S. Emmott, A. G. Smith, Science (New York, NY) 344, 1156 (2014).

7. J. R. Karr, et al., Cell 150, 389 (2012).

8. A. C. Babtie, P. Kirk, M. P. H. Stumpf, Proceedings Of The National Academy Of Sciences Of The United States Of America 111, 18507 (2014).

9. P. Meyer, et al., BMC systems biology 8, 13 (2014).

10. A. F. Villaverde, et al., Computer methods and programs in biomedicine 119, 17 (2015).

11. J. Ruess, A. Milias-Argeitis, J. Lygeros, Journal Of The Royal Society Interface 10, 20130588 (2013).

12. M. Sunnåker, et al., Science signaling 6, ra41 (2013).

13. D. Silk, P. D. W. Kirk, C. P. Barnes, T. Toni, M. P. H. Stumpf, PLoS computational biology 10, e1003650 (2014).

14. J. Liepe, et al., eLife 4 (2015).

15. J. E. Cohen, PLoS biology 2, e439 (2004). 